www.jmscr.igmpublication.org

Impact Factor 5.244

Index Copernicus Value: 83.27

ISSN (e)-2347-176x ISSN (p) 2455-0450

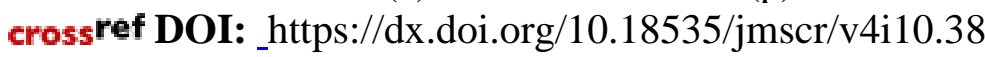

Journal Of Medical Science And Clinical Research

\title{
Proximal Epithelioid Sarcoma: A Diagnostic Challenge
}

\author{
Authors \\ Dr Ajeet Kumar ${ }^{1}$, Dr Guddi Rani Singh ${ }^{2}$, Dr V.Choudhary ${ }^{3}$, \\ Dr Suryajit Kumar Singh ${ }^{4}$, Dr Amit Kumar Sinha ${ }^{5}$ \\ ${ }^{1,2,4,5}$ Senior Resident, Department of Pathology, IGIMS, Patna ${ }^{3}$ Additional Professor \\ Department of Pathology, IGIMS, Patna, Bihar
}

\begin{abstract}
Epithelioid sarcoma is an uncommon slow-growing soft tissue malignancy, associated with a high incidence of local recurrence and metastasis. We report a 30-year-old male presented with huge mass in left thigh with difficulty in walking, clinically misdiagnosed as clear cell sarcoma. Multiple nodule noted in left lung parenchyma suggestive of metastasis. Later histopathological examination and IHC confirmed the diagnosis of epithelioid sarcoma. Here we present the case because of large number of histological mimics of epithelioid sarcoma and its unusual location.

Keywords: Epithelioid sarcoma, Soft tissue sarcoma, metastasis.
\end{abstract}

\section{Introduction}

Epithelioid sarcoma, first described by Enzinger in 1970, is a rare soft tissue sarcoma of adolescents and young adults usually involving the distal part of upper extremities ${ }^{[1,2]}$. The epithelioid sarcomas are difficult to diagnose and can be confused with a variety of malignant and benign conditions; especially granulomatous process, synovial sarcoma and ulcerating squamous cell carcinoma. This is usually a slow growing tumour which commonly involves the dermal or subcutaneous area of distal part of upper extremity. The "proximal type or axial type" variant was later recognized in $1997^{[2]}$, with very few reports on such cases. It is usually seen in adults and are commonly deep seated and occurs in perineum, sex organs, pelvis and thighs. It is more aggressive with poor prognosis with tendency for local recurrence and early metastases. The proximal type epithelioid sarcomas are rare undifferentiated soft tissue sarcomas usually seen in adults with epithelioid features and a frequent rhabdoid phenotype on histopathology thus posing diagnostic difficulty and has to be differentiated from extrarenal rhabdoid tumour, synovial sarcoma, angiosarcoma and melanoma ${ }^{[1,3]}$.

\section{Case Report}

A 30-year-old male presented with swelling over his left thigh. His complain started as difficulty in walking 4 yrs ago. Then he noted a small nodule like swelling in left thigh, which slowly progressed in size. He had consulted local quackes but the size of swelling continued to increase. On clinical examination, he was thin built and condition of his health was not well. There was a huge swelling of size $6 \times 3 \times 3 \mathrm{~cm}$ on left thigh. The 


\section{JMSCR Vol||04||Issue ||10||Page 13133-13137||October}

swelling was subcutaneous and freely mobile.. Xray of the thigh revealed no bony involvement (Fig no.1 a.). MRI, left thigh shows ill defined lobulated mass lesion involving the posterior compartment of mid thigh.(Fig. 1b,1c.).

CT Scan of thorax revealed multiple nodule in left lung parenchyma -suggestive of metastasis. (Fig no.1d).. NCCT of brain revealed unremarkable picture.

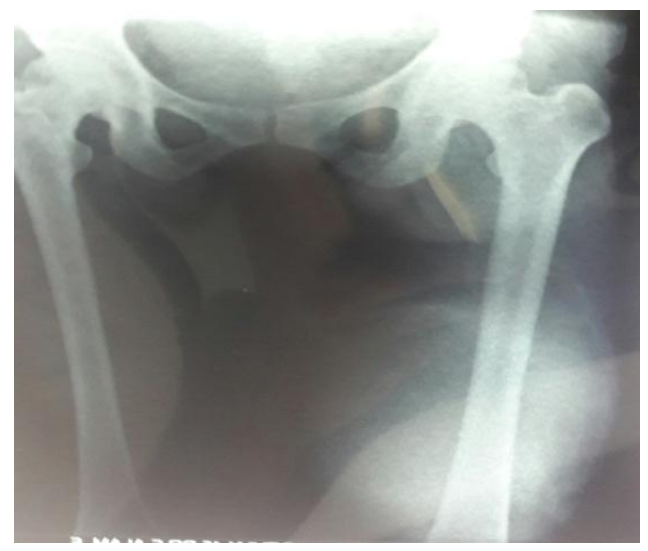

Fig- 1a

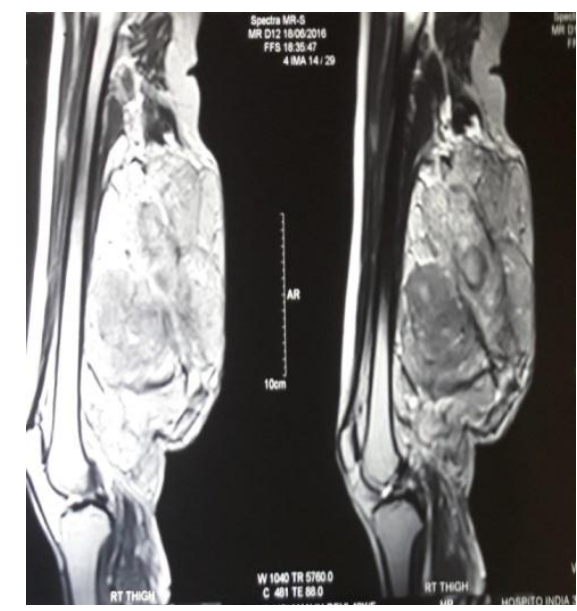

Fig- 1b

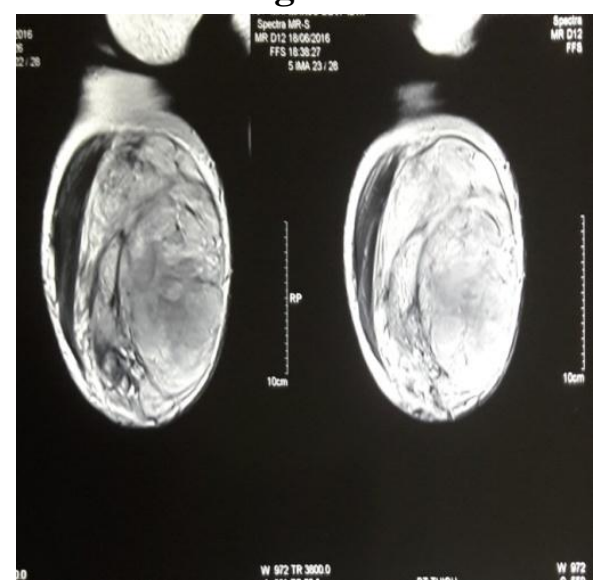

Fig-1c

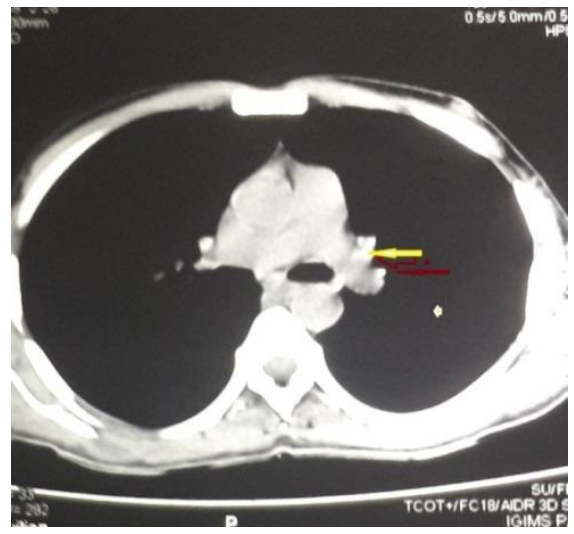

Fig- 1d

A biopsy from the swelling microscopically revealed a cellular tumour composed of interlacing bundles of moderate to highly pleomorphic oval to spindle shaped cells with hardly any intervening stroma. Individual cells shows eccentric oval nuclei with moderate amount of deep eosinophilic cytoplasm.(Fig 2a). Area of necrosis and few mitosis were seen.On Immunohistochemical staining-CK, and Vimentin positive in majority of tumor cells Fig $(2 b, 2 c)$. .CD34 and CD99 positive in several tumor cells (Fig 2d 2e). Desmin and HMB-45( Fig 2f) were negative. Ki-67 was $50 \%$ positive.

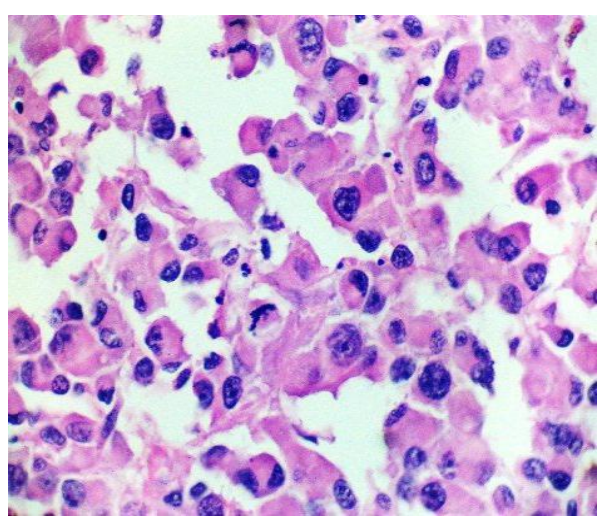

Fig 2a- H\&E

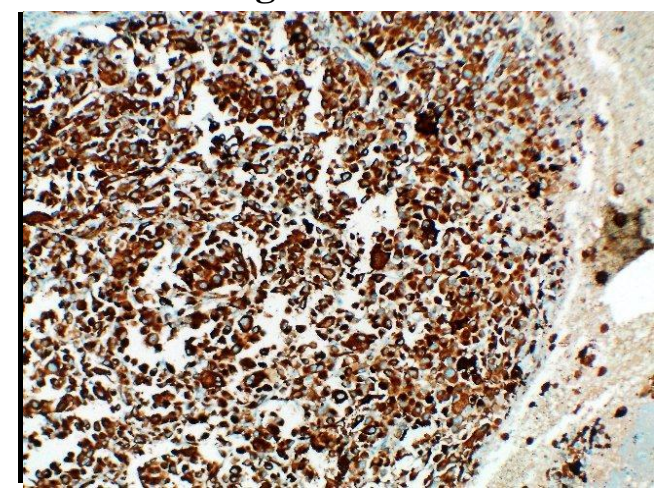

Fig 2b- CK Positive 


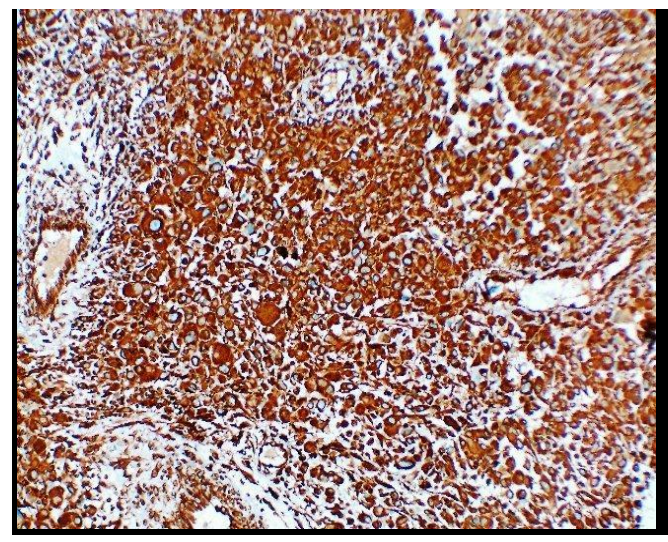

Fig 2c-Vimentin Positive

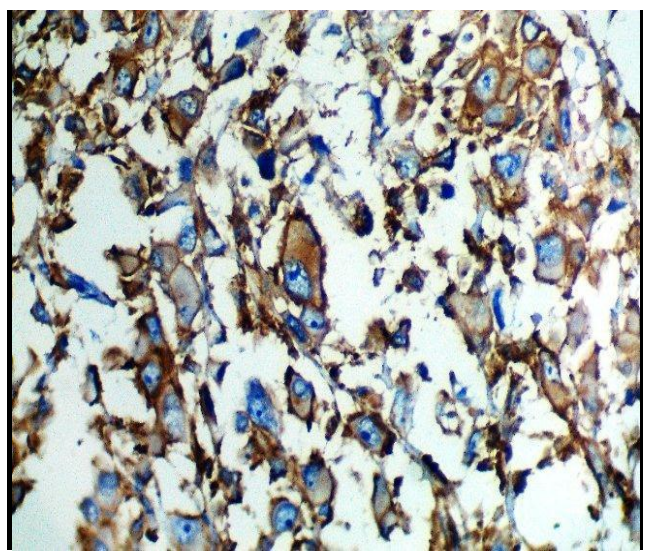

Fig 2d-CD 34 Positive

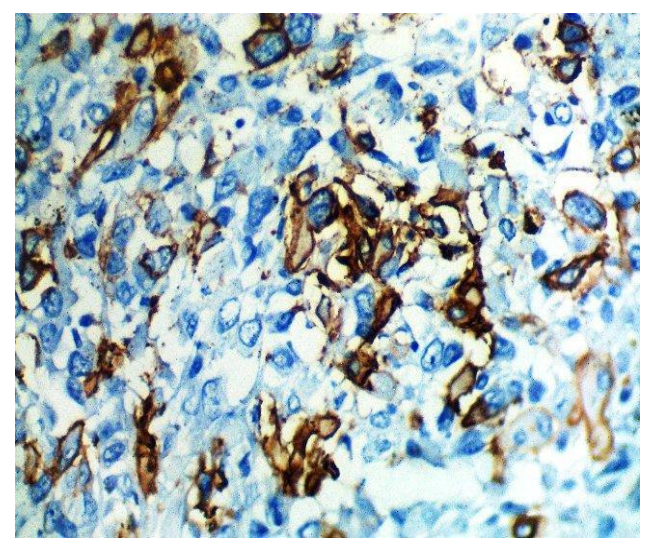

Fig 2e-CD99 Positive

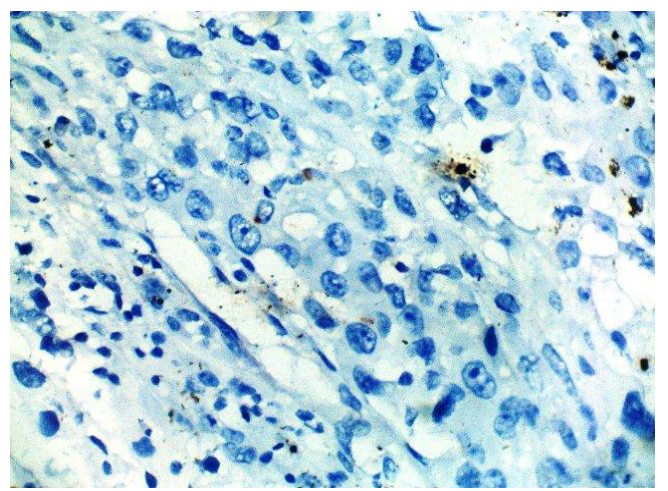

Fig 2f-Desmin, HMB 45 Negative
Complete wide local excision was done, followed by adjuvant local radiotherapy, in an attempt to lower the risk of local recurrence. Chemotherapy was done for metastasis to the lung, with a standard regimen, which typically includes ifosfamide or doxorubicin, sometimes in combination. Now the patient is free of the disease one year after the diagnosis.

\section{Discussion}

Epithelioid sarcoma is a rare soft tissue sarcoma arising from mesenchymal tissue and characterized by epithelioid- like features. It accounts for less than $1 \%$ of all soft tissue sarcomas. It commonly presents itself in distal limbs (fingers, hands, forearms, or feet) of young adults (yet no age group is immune) as a small, soft mass or a series of bumps. A proximal version has also been described, frequently occurring in the upper extremities ${ }^{(2)}$.

Microscopically, ES is usually multinodular with a central necrosis surrounded by bland polygonal cells with eosinophilic cytoplasm and peripheral spindling. The classic-type ES has cells with only mild atypia. Therefore, it has many histopathological mimics,including benign and malignant conditions, such as chronic granulomatous inflammation (especially rheumatoid nodules), clear-cell sarcoma of the tendon and aponeurosis (amelanotic melanoma of soft part), schwannoma, metastatic squamous cell carcinoma, metastatic adenocarcinoma (especially renal cell carcinoma), synovial sarcoma, epithelioid hemangioendothelioma, epithelioid leiomyosarcoma, and malignant extrarenal rhabdoid tumors of the soft tissue $^{\cdot(1,4-16)}$. Therefore ES is characterized by diagnostic difficulties, both clinically and histopathologically, which result in a high frequency of initial misdiagnosis and the loss of crucial treatment time. However, the presence of mitotic activity should raise the index of suspicion for ES, and the presence of diffuse epithelial marker positivity indicates the correct diagnosis The proximal type or axial type is an aggresive variant of ES. Histologically, the proximal 
subtype differs from the classic type by its larger epithelioid cells, with vesicular nuclei,and prominent nucleoli; copious, eccentric cytoplasm; marked cytologic atypia; and frequent rhabdoid features. Tumoral necrosis, frequent mitosis, vascular invasion, and absence of a granulomatous appearance are common finding in the proximal subtype ${ }^{(8,9)}$. Clinically, the proximal subtype differs from the classic type by its multinodular growth pattern, more frequent occurrence in older patients, more deep-seated location ${ }^{(8)}$. Proximaltype ES should also be distinguished from other neoplasms with epithelioid and/or rhabdoid features, such as malignant extrarenal rhabdoid tumor, epithelioid rhabdomyosarcoma, melanoma, malignant peripheral nerve sheath tumor, and undifferentiated carcinoma ${ }^{(8,9,12)}$. Appropriate immunohistochemical studies showing positivity for vimentin, cytokeratins, S-100, CD99, CD34, epithelial membrane antigen and negativity for the Desmin, SMA, HMB 45 are helpful to exclude most of these other mentioned tumors from the differential diagnosis. Coexpression of vimentin and keratin is thought to be characteristic of ES.(1,4-16).Carcinomas, both primary (especially those of adnexal origin) and metastatic, can have a presentation similar to ES. Carcinomas are nearly always CD34 negative, and although CD34 is only expressed in about $50 \%$ to $60 \%$ of ES, this marker is particularly useful for diagnosing the deep-seated, proximal-type ES, which can be misdiagnosed as metastatic carcinoma of an unknown primary site. ${ }^{(4,7)}$. Additionally, metastatic renal cell carcinoma may be delineated by virtue of its glandular and papillary pattern despite the similarity in cell morphology. Synovial sarcomas may have cytokeratin and vimentin positivity; however, the tumor cells are usually less pleomorphic, are oval to spindled, and often show areas of glandular differentiation, unlike ES. Synovial sarcoma can have plump epithelioid and even rhabdoid cells on occasion. The immuneprofile of the synovial sarcoma and ES can overlap, but synovial sarcoma is often positive for S100 protein and only very rarely CD34 positive.
Epithelioid vascular tumors can resemble ES, and additionally, some express CK. However, they differ from ES in being positive for CD31, factor VIIIa, and FLI-1 but negative for cD34. ${ }^{(4,7)}$. Rhabdomyosarcoma is readily excluded by immunehistochemistry, showing positive epithelial markers and and absence of desmin ${ }^{(4,7)}$.It might not be possible to distinguish S100 proteinnegative rhabdoid melanoma from proximal-type ES, especially as some of the latter have been reported to be HMB-45 positive ${ }^{(5,8)}$. Many tumors formerly diagnosed as malignant rhabdoid tumor are now classified as proximal-type ES. FanburgSmith et $\mathrm{al}^{(16)}$ reported that all extrarenal rhabdoid tumorr are negative for CD34. Therefore, ES and extrarenal rhabdoid tumor are considered separate entities.

A local recurrence rate of $35 \%,{ }^{(7)}, 69 \%{ }^{(2)}$ and $77 \%$ ${ }^{(5)}$ has been reported in the literature. Epitheloid sarcoma has a tendency to be multifocal at recurrence. The frequency of regional lymph node metastasis is typically between 22 and $45 \%$. Distant metastasis has been reported in up to $45 \%$ of patients with epithelioid sarcoma ${ }^{(1,2)}$ The metastasis is usually to the lung ,pleura, bone, brain, and other locations, including the $\operatorname{scalp}^{(8)}$. Metastasis was detected in left lung in our patient. The treatment of primary disease is wide local excision, followed by adjuvant radiotherapy, in an attempt to lower the risk of local recurrence. Though amputation is required relatively frequently in epithelioid sarcoma because of the tendency of multifocal disease, there is no survival advantage from primary amputation. Chemotherapy is recommended for metastatic disease, with a standard regimen, which typically includes ifosfamide or doxorubicin, sometimes in combination. Our patient underwent wide excision of the lesion followed by radiotherapy and is free of the disease one year after the diagnosis.

\section{Conclusion}

To conclude we would like to emphasize that it is important to diagnose this rare variety of soft tissue sarcoma as early as possible due to better 
patient outcome in absence of metastases and smaller size of the tumour. Due to inconsistent pathologic features of epithelioid sarcoma, it is of utmost importance that the biopsy and interpretation of the microscopic specimen is done by an experienced musculoskeletal oncologist and pathologist, respectively.

\section{References}

1. Enzinger, F. M. (1970). "Epithelioid sarcoma. A sarcoma simulating a granuloma or a carcinoma". Cancer. 26 (5): 1029-41.

2. Guillou, L; Wadden, C; Coindre, JM; Krausz, T; Fletcher, CD (1997). "'Proximal-type' epithelioid sarcoma, a distinctive aggressive neoplasm showing rhabdoid features. Clinicopathologic, immunohistochemical, and ultrastructural study of a series". The American Journal of Surgical Pathology. 21 (2): 130-46.

3. Fisher C. Epithelioid sarcoma of Enzinger. Adv Anat Pathol.2006;13:114-121.

4. Miettinen M, Fanburg-Smith JC, Virolainen M, Shmookler BM, Fetsch JF.7 Epithelioid sarcoma: an immunohistochemical analysis of 112 classical and variant cases and a discussion of the differential diagnosis.Hum Pathol.1999;30 :934-942.

5. Chase DR, Enzinger FM. Epithelioid sarcoma: diagnosis, prognostic indicators, and treatment.Am J Surg Pathol.1985; 9:241-260.

6. Evans HL, Baer SC. Epithelioid sarcoma: a clinicopathologic and prognosticstudy of 26 cases. Semin Diagn Pathol.1993; 10:286-89.

7. Spillane AJ, Thomas JM, Fisher C. Epithelioid sarcoma: the clinicopathological complexities of this rare soft tissue sarcoma. Ann Surg Oncol.2000;7:218225-263

8. Guillou L, Wadden C, Coindre JM, Krausz T, Fletcher CD. 'Proximal-type', epithet- lioid sarcoma, a distinctive aggressive neoplasm showing rhabdoid features: clinicopathologic, immunohistochemical, and ultrastructural study of a series.Am J Surg Pathol.1997;21:130-9.

9. Proximal-type epithelioid sarcoma: a clinicopathologic study of 20 cases. MoPathol.2001;14:655-663

10. Sur M, Nayler SJ. Proximal epithelioid sarcoma-a misnomer Histopathology 2001;39:641-643.

11. Lee HI, Kang KH, Cho YM, Lee OJ, Ro JY. Proximal-type epithelioid sarcoma with elevated serum CA 125: report of a case with CA

12. 25 immunoreactivity. Arch Pathol Lab Med.2006;130:871-874.

13. Kato H, Hatori M, Kokubun $\mathrm{S}$, et al. CA125 expression in epithelioid sarcoma. Jpn J Clin Oncol.2004;34:149-154.

14. Kato H, Hatori M, Watanabe M, Kokubun S. Epithelioid sarcomas with elevated serum CA125: report of two cases.Jpn J Clin Oncol.2003;33:141-144.

15. Fanburg-Smith JC, Hengge M, Hengge UR, Smith JS Jr, Miettinen M. Extrarenal rhabdoid tumors of soft tissue: a clinicopathologic and immunochemical study of 18 cases Ann Diagn Pathol. 1998;2:351-362. 